\title{
O cuidador do portador de Alzheimer: revisão integrativa sobre o cuidar e a sobrecarga da atividade
}

\author{
The caregiver of the Alzheimer carrier: narrative review on the care and the burden of the \\ activity \\ El cuidador del portador Alzheimer: revisión narrativa sobre el cuidado y la sobrecarga de \\ la actividad
}

Maira Beatrine da Rocha Uchôa ${ }^{1 *}$, Marlucilena Pinheiro da Silva ${ }^{1}$, Darci Francisco dos Santos Junior $^{1}$, Victor Hugo Oliveira Brito ${ }^{1}$, Fabio Rodrigues Trindade ${ }^{2}$.

\section{RESUMO}

Objetivo: Destacar o que a literatura atualmente disponível discorre sobre o cuidador do portador de Alzheimer e a sobrecarga dessa atividade, apresentando os resultados por meio de uma revisão integrativa das publicações e discuti-las. Métodos: Trata-se de um estudo qualitativo do tipo revisão integrativa de literatura, com busca dos estudos no período de 2006 a 2019 nas bases eletrônicas: LILACS, BVS, SCIELO e Research Gate, e se utilizou os descritores: Cuidador, Idoso e Alzheimer. Resultados: Foram identificados 23 artigos nacionais, sendo 19 artigos originais e 4 de revisão, que caracterizam as atividades do cuidador que vão desde o auxílio na higiene a tarefas externas, o que evidencia a sobrecarga relacionada a rotina de cuidar do portador de Alzheimer, e consequentemente, no adoecimento. Considerações finais: Os estudos apontam intensa sobrecarga do cuidador, o que repercute negativamente na sua saúde mental e física e a necessidade de cuidados em saúde para esta população.

Palavras-chave: Cuidador, Alzheimer, Idoso.

\section{ABSTRACT}

Objective: To highlight what the currently available literature discusses about the caregiver of Alzheimer's patients and the overload of this activity, presenting the results through an integrative review of publications and discussing them. Methods: This is a qualitative study of the type integrative literature review, with a search for studies from 2006 to 2019 in the electronic databases: LILACS, BVS, SCIELO and Research Gate, and the descriptors: Caregiver, Elderly and Alzheimer were used. Results: 23 national articles were identified, 19 of which were original and 4 review articles, which characterize the caregiver's activities, ranging from hygiene assistance to external tasks, which shows the burden related to the routine of caring for Alzheimer's patients, and consequently, in illness. Final considerations: The studies show an intense burden on the caregiver, which negatively affects their mental and physical health and the need for health care for this population.

Key words: Caregiver, Alzheimer, Elderly.

\section{RESUMEN}

Objetivo: Resaltar lo que la literatura actualmente disponible discute sobre el cuidador de pacientes con Alzheimer y la sobrecarga de esta actividad, presentando los resultados a través de una revisión integradora de publicaciones y discutiéndolos. Métodos: Este es un estudio cualitativo de la revisión de literatura integrativa tipo, con una búsqueda de estudios de 2006 a 2019 en las bases de datos electrónicas: LILACS,

\footnotetext{
${ }^{1}$ Universidade Federal do Amapá (UNIFAP), Macapá - AP. `E-mail: mairauch91@gmail.com

2 Universidade Federal do Piauí (UFPI), Teresina - PI.
} 
BVS, SCIELO y Research Gate, y los descriptores: Cuidador, Ancianos y Alzheimer. Resultados: Se identificaron 23 artículos nacionales, 19 de los cuales eran originales y 4 artículos de revisión, que caracterizan las actividades del cuidador, que van desde asistencia de higiene hasta tareas externas, lo que resalta la carga relacionada con la rutina del cuidado de pacientes con Alzheimer, y en consecuencia, en la enfermedad. Consideraciones finales: Los estudios muestran una carga intensa sobre el cuidador, que afecta negativamente su salud mental y física y la necesidad de atención médica para esta población.

Palabras clave: Cuidador, Alzheimer, Anciano.

\section{INTRODUÇÃO}

A população mundial está envelhecendo. O Relatório da Organização das Nações Unidas (ONU) confirma que estamos envelhecendo devido ao aumento da expectativa de vida e à queda dos níveis de fertilidade. Segundo o documento, até 2050, uma em cada seis pessoas no mundo terá mais de 65 anos, o equivalente a $16 \%$ da população (ONU - WPP, 2019). O Brasil, seguindo a tendência de envelhecimento mundial, ganhou 4,8 milhões de idosos desde 2012, alcançando 30,2 milhões de idosos em 2017, segundo a Pesquisa Nacional por Amostra de Domicílios Contínua - Características dos Moradores e Domicílios (IBGE, 2017).

Diante deste cenário da estimativa de vida mais elevada, presenciamos uma modificação das doenças e agravos mais prevalentes, que atualmente, são crônicos e não transmissíveis. No Brasil, estima-se que $85 \%$ dos idosos apresentam pelo menos uma doença crônica, e destes, no mínimo, 10\% com comorbidades (LEITE BS, et al., 2017a; ARAKAKI BK, et al., 2012). Segundo dados do Relatório Mundial da Doença de Alzheimer, da Federação Alzheimer's Disease International (ADI), cerca de 47 milhões de pessoas vivem com demência em todo o mundo. Este número está previsto para aumentar para mais de 131,5 milhões no ano de 2050. Além disso, o número estimado de pessoas com Doença de Alzheimer (DA) chega a 35,6 milhões em todo o mundo, sendo considerada pela Organização Mundial de Saúde (OMS), como a forma mais comum de demência que atinge os idosos, sendo responsável por aproximadamente 60 a $70 \%$ dos casos.

No Brasil, pela inexistência de pesquisa nacional específica, não há dados oficiais a respeito do número de pessoas com doença de Alzheimer, porém, segundo os dados do Instituto Brasileiro de Geografia e Estatística (IBGE/PNAD 2017) estima-se que existam 1,2 milhão de pacientes com DA no país e uma incidência de cerca de 100 mil novos casos a cada ano (CHAIMOWICZ F, 2013; GARCIA CR, et al., 2017; GRATÃO ACM, et al., 2013; SILVA MIS, et al., 2018). Tal patologia é caracterizada como crônica neurodegenerativa, ou seja, causa disfunções cerebrais que são responsáveis pelo comprometimento cognitivo e funcional nos idosos. À medida que a doença evolui, o comprometimento cerebral também aumenta, deixando os portadores em situação vulnerável e de dependência, além de afetar o funcionamento ocupacional e social de cada indivíduo (COSTA TG, et al., 2011).

Ao falar de demência, necessariamente a dependência surge como consequência da doença. Dessa forma, o idoso que apresenta um potencial favorável à perda das capacidades funcionais e/ou mentais, necessita de maiores cuidados, que são assumidos por cuidadores informais familiares do doente, ou por cuidadores formais, profissionais remunerados que atuam no cuidar de indivíduos portadores de doenças crônicas e/ou em estado de vulnerabilidade (DELALIBERA M, et al., 2018; LEITE BS, et al., 2017a; MAIA JM, et al., 2018). Quem cuida, o chamado cuidador, tem um papel que exige a tomada de decisões e a incorporação de atividades que passam a ser de sua inteira responsabilidade (XIMENES MA, et al.,2014).

Perante a situação apresentada, questiona-se, o que a literatura descreve sobre o cuidador e a sobrecarga do cuidador do portador de Alzheimer? Quais as dificuldades vivenciadas por este cuidador? O cuidador é sobrecarregado em sua função?

Para responder à pergunta apresentada têm-se como objetivo caracterizar o que a literatura indica sobre o cuidador e a sobrecarga do cuidador do portador de Alzheimer, portanto, o limitando número de 
investigações existentes na literatura sobre esta temática e apresentar os resultados através de uma revisão narrativa das publicações e discuti-las.

\section{MÉTODOS}

Trata-se de um estudo qualitativo de revisão integrativa de literatura, apropriada para discutir o estado da arte de um determinado assunto, sob o ponto de vista teórico ou contextual. É constituída de análise da literatura publicada em livros, artigos de revistas impressas e/ou eletrônicas, com interpretação e análise crítica pessoal do autor. Esta categoria de artigo é fundamental para a aquisição e atualização do conhecimento sobre uma temática específica, evidenciando novas ideias, métodos e subtemas que têm recebido maior ou menor ênfase na literatura selecionada (ELIAS CSR, et al., 2012).

A busca dos estudos foi realizada nos meses de janeiro a agosto de 2019 nas bases eletrônicas: Literatura Latino-Americana e do Caribe em Ciências da Saúde (LILACS), Biblioteca Virtual de Saúde (BVS), Scientific Eletronic Library Online (SCIELO), no portal de periódicos ResearchGate, no portal de periódicos da UFPR e da USP. Foram utilizados os seguintes descritores padronizados pelos Descritores em Ciências da Saúde (DeCS): Cuidador, Alzheimer e Idoso. Foram incluídos artigos disponibilizados na íntegra (freefulltext), nos últimos 13 anos (2006 a 2019), nos idiomas português, inglês e espanhol, e que tratassem da temática: sobrecarga do cuidador do portador de Alzheimer.

Os títulos e resumos das publicações foram revisados e analisados. Das 422 referências obtidas nas bases de dados mencionadas, 68 artigos foram selecionados após a leitura de títulos e resumos que se adequavam a temática da pesquisa. Foram excluídos teses e dissertações e artigos que não se adequavam a temática da pesquisa. Posteriormente, todos os artigos selecionados foram lidos na íntegra para análise das características gerais, tais como ano de publicação, objetivos, metodologia, resultados obtidos e discussão, para refinamento e então 23 foram elegíveis e incluídos neste trabalho, sendo 19 artigos originais e 4 artigos de revisão.

\section{RESULTADOS E DISCUSSÃO}

Dos 23 artigos incluídos neste trabalho, distribuídos nas seguintes bases: 3 (LILACS), 5 (BVS), 12 (SCIELO), 1 (REDALYC), 1 (RESEARCHGATE), 1 (PERIÓDICOS USP), 1 (PERÍODOICOS UFPR), todos nacionais, sendo 19 artigos originais e 4 artigos de revisão, com predomínio de publicações entre os anos 2012 e 2017 (Gráfico 1).

Gráfico 1 - Quantificação de artigos pelo ano de publicação de 2006 a 2019.

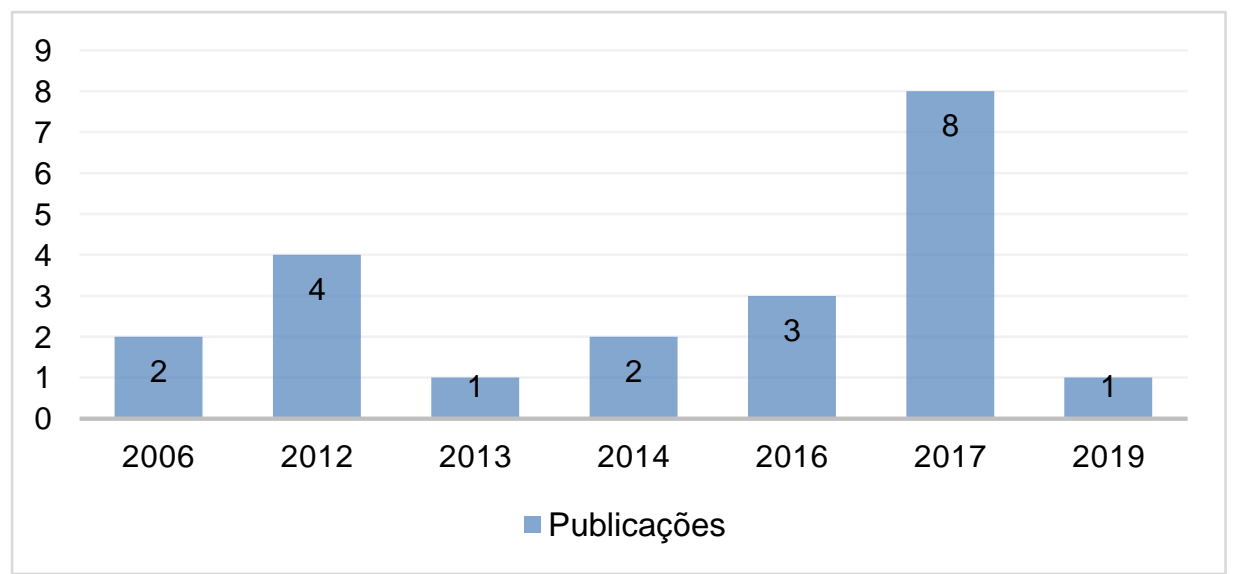

Fonte: Uchôa MBR, et al., 2019.

Para melhor esclarecimento, os artigos selecionados foram organizados em um quadro, no qual foram apresentados os achados, descritos segundo seus respectivos títulos e autorias dos estudos, periódicos, ano de publicação e base de dados em que foram encontrados (Tabela 1). 
Tabela 1 - Catalogação dos artigos por autoria e pelo Título, Periódico e Base de dados.

\begin{tabular}{|c|c|}
\hline Autoria, Ano e Tipo de Artigo & Título, Periódico e Base de dados \\
\hline $\begin{array}{l}\text { ALMEIDA LPB, et al., } 2018 \\
\text { (Artigo Original) }\end{array}$ & $\begin{array}{l}\text { Características sociais e demográficas de idosos cuidadores e motivos para cuidar da } \\
\text { pessoa idosa em domicílio. REME rev. min. Enferm. } \\
\text { LILACS }\end{array}$ \\
\hline $\begin{array}{l}\text { ARAÚJO CLO, et al., } 2012 \\
\text { (Artigo Original) }\end{array}$ & $\begin{array}{l}\text { Perfil de cuidadores de idosos com Doença de Alzheimer. Revista Kairós Gerontologia. } \\
\text { SCIELO }\end{array}$ \\
\hline $\begin{array}{l}\text { ARAKAKI BK, et al., } 2012 \\
\text { (Artigo Original) }\end{array}$ & $\begin{array}{l}\text { Análise do desgaste e da sobrecarga de cuidadores/familiares de idosos com doença de } \\
\text { Alzheimer causado pelos sintomas psicológicos e comportamentais. Revista de Terapia } \\
\text { Ocupacional da Universidade de São Paulo. } \\
\text { Periódicos USP }\end{array}$ \\
\hline $\begin{array}{l}\text { BAPTISTA BO, et al., } 2012 \\
\text { (Artigo de Revisão) }\end{array}$ & $\begin{array}{l}\text { A sobrecarga do familiar cuidador no âmbito domiciliar: uma revisão integrativa da } \\
\text { literatura. Revista Gaúcha de Enfermagem. }\end{array}$ \\
\hline $\begin{array}{l}\text { BRIGOLA AG, et al., } 2017 \\
\text { (Artigo Original) }\end{array}$ & $\begin{array}{l}\text { Perfil de saúde de cuidadores familiares de idosos e sua relação com variáveis do cuidado: } \\
\text { um estudo no contexto rural. Revista Brasileira de Geriatria e Gerontologia } \\
\text { SCIELO }\end{array}$ \\
\hline $\begin{array}{l}\text { CARDOSO VB, et al., } 2017 \\
\text { (Artigo de Revisão) }\end{array}$ & $\begin{array}{l}\text { A doença de Alzheimer em idosos e as consequências para cuidadores domiciliares. } \\
\text { Memorialidades. } \\
\text { SCIELO }\end{array}$ \\
\hline $\begin{array}{l}\text { GARCIA CR, et al., } 2017 \\
\text { (Artigo Original) }\end{array}$ & $\begin{array}{l}\text { Cuidadores familiares de idosos com a doença de Alzheimer. Revista Kairós } \\
\text { Gerontologia. } \\
\text { SCIELO }\end{array}$ \\
\hline $\begin{array}{l}\text { GRATÃO ACM, et al, } 2013 \\
\text { (Artigo Original) }\end{array}$ & $\begin{array}{l}\text { Dependência funcional de idosos e a sobrecarga do cuidador. RevEscEnfermUSP. } \\
\text { SCIELO }\end{array}$ \\
\hline $\begin{array}{l}\text { GUERRA HS, et al., } 2017 \\
\text { (Artigo Original) }\end{array}$ & $\begin{array}{l}\text { A sobrecarga do cuidador domiciliar. Revista Brasileira em promoção da Saúde. } \\
\text { REDALYC }\end{array}$ \\
\hline $\begin{array}{l}\text { GUTIERREZ LLP, et al., } 2017 \\
\text { (Artigo Original) }\end{array}$ & $\begin{array}{l}\text { Caracterização de cuidadores de idosos da região metropolitana de Porto Alegre (RS): } \\
\text { perfil do cuidado. Rev Saúde Debate. } \\
\text { SCIELO }\end{array}$ \\
\hline $\begin{array}{l}\text { KUCMANSKI LS, et al., } 2016 \\
\text { (Artigo Original) }\end{array}$ & $\begin{array}{l}\text { Doença de Alzheimer: desafios enfrentados pelo cuidador no cotidiano Familiar. Rev. Bras. } \\
\text { Geriatr. Gerontol. } \\
\text { SCIELO }\end{array}$ \\
\hline $\begin{array}{l}\text { LEITE BS, et al., } 2017 a \\
\text { (Artigo Original) }\end{array}$ & $\begin{array}{l}\text { Relação do Perfil Epidemiológico dos Cuidadores De Idosos Com Demência a a } \\
\text { Sobrecarga do Cuidado. Cogitare Enfermagem. } \\
\text { PERIÓDICOS UFPR }\end{array}$ \\
\hline
\end{tabular}




\begin{tabular}{|c|c|}
\hline Autoria, Ano e Tipo de Artigo & Título, Periódico e Base de dados \\
\hline $\begin{array}{l}\text { LEITE BS, et al., } 2017 \mathrm{~b} \\
\text { (Artigo Original) }\end{array}$ & $\begin{array}{l}\text { A vulnerabilidade dos cuidadores de idosos com demência: estudo descritivo transversal. } \\
\text { Revista Brasileira de Enfermagem. } \\
\text { SCIELO }\end{array}$ \\
\hline LEMOS ND, et al., 2006 (Artigo Original) & $\begin{array}{l}\text { Cuidando do paciente com Alzheimer: o impacto da doença no cuidador. Saúde Soc. } \\
\text { SCIELO }\end{array}$ \\
\hline $\begin{array}{l}\text { LINO VTS, et al., } 2016 \\
\text { (Artigo Original) }\end{array}$ & $\begin{array}{l}\text { Prevalência de sobrecarga e respectivos fatores associados em cuidadores de idosos } \\
\text { dependentes, em uma região pobre do Rio de Janeiro, Brasil.Cad. Saúde Pública. } \\
\text { SCIELO }\end{array}$ \\
\hline $\begin{array}{l}\text { LUZARDO AR, et al., } 2006 \\
\text { (Artigo Original) }\end{array}$ & $\begin{array}{l}\text { Características de idosos com Doença de Alzheimer e seus cuidadores: uma série de } \\
\text { casos em um serviço de neurogeriatria. Revista Texto Contexto Enfermagem. } \\
\text { BVS }\end{array}$ \\
\hline MARINS AMF e SILVA J., 2017 (Artigo de Revisão) & $\begin{array}{l}\text { O impacto do comportamento do idoso com doença de Alzheimer na vida do cuidador. } \\
\text { Revista de Enfermagem do Centro-Oeste Mineiro. } \\
\text { LILACS }\end{array}$ \\
\hline $\begin{array}{l}\text { MENDES PN, et al., } 2019 \\
\text { (Artigo Original) }\end{array}$ & $\begin{array}{l}\text { Sobrecargas física, emocional e social dos cuidadores informais de idosos. Acta paul. } \\
\text { enferm. } \\
\text { BVS }\end{array}$ \\
\hline $\begin{array}{l}\text { MUNIZ EA, et al., } 2016 \\
\text { (Artigo Original) }\end{array}$ & $\begin{array}{l}\text { Grau de sobrecarga dos cuidadores de idosos atendidos em domicílio pela Estratégia } \\
\text { Saúde da Família. RevSaude Debate. } \\
\text { BVS }\end{array}$ \\
\hline $\begin{array}{l}\text { NARDI EFR, et al., } 2012 \\
\text { (Artigo Original) }\end{array}$ & $\begin{array}{l}\text { Dificuldades dos cuidadores familiares no cuidar de um idoso dependente no domicílio. } \\
\text { Cienc cuid saúde. } \\
\text { BIREME }\end{array}$ \\
\hline $\begin{array}{l}\text { SEIMA MD, et al., } 2014 \\
\text { (Artigo Original) }\end{array}$ & $\begin{array}{l}\text { Relação no cuidado entre o cuidador familiar e o idoso com Alzheimer. Revista Brasileira } \\
\text { de Enfermagem. } \\
\text { SCIELO }\end{array}$ \\
\hline $\begin{array}{l}\text { SILVA MIS, et al., } 2018 \\
\text { (Artigo Original) }\end{array}$ & $\begin{array}{l}\text { Doença de Alzheimer: repercussões biopsicossociais na vida do cuidador familiar. Revista } \\
\text { de Enfermagem UFPE On Line. } \\
\text { BVS }\end{array}$ \\
\hline $\begin{array}{l}\text { XIMENES MA, et al.., } 2014 \\
\text { (Artigo de Revisão) }\end{array}$ & $\begin{array}{l}\text { Doença de Alzheimer: a dependência e o cuidado. Revista Kairós Gerontologia. } \\
\text { BVS }\end{array}$ \\
\hline
\end{tabular}

Doença de Alzheimer: a dependência e o cuidado. Revista Kairós Gerontologia.

Fonte: Uchôa MBR, et al., 2019. 
A partir da leitura criteriosa dos textos selecionados, foi possível observar que os estudos são unânimes no que concerne à contextualização inicial da temática, a doença de Alzheimer causa importante impacto ou mudanças na vida do indivíduo que vivencia o processo demencial, e, sobretudo, alterações importantes na vida do cuidador, especialmente, o familiar. Essas alterações trazem sofrimento para as famílias cuidadoras, além cansaço e sobrecarga para o cuidador. Nesse sentido, pode-se apontar as atividades extensas e longa jornada de cuidado ao idoso com Alzheimer interferem e/ou causam impactos na vida do cuidador, resultando em sobrecarga física e/ou emocional.

Tais atividades relacionadas ao cuidado e a caracterização da sobrecarga serão discorridos a seguir. De modo a organizar o produto do levantamento de artigos para melhor visualização dos achados, optou-se por reuni-los em duas categorias que respondam os questionamentos deste estudo: 1) O Cuidador e Cuidar do Portador de Alzheimer, e 2) A Sobrecarga do Cuidador do Portador Alzheimer.

\section{O Cuidador e o Cuidar do Portador de Alzheimer}

Quem assume o papel de cuidar - o chamado Cuidador - passa a ter um indivíduo por sua inteira responsabilidade, que exige a tomada de decisões e a incorporação de atividades externas a sua rotina até exaustivas, que incluem organização desde a organização com os cuidados domiciliares ao indivíduo com Alzheimer, até acompanhamento em consultas, exames, controle financeiro e o abastecimento do lar. Todas essas atribuições podem repercutir de forma negativa na vida do cuidador, sendo esse grupo merecedor de atenção, orientação e preocupação dos profissionais e serviços de saúde. Em países como o Brasil, 90\% dos cuidadores são informais, ou seja, membros da família que não possuem recursos financeiros para manter um apoio formal (KUCMANSKI LS, et al.,2016; LEITE BS, et al., 2017b).

Normalmente, os cuidadores oferecem cuidados a qualquer indivíduo em situação de doença ou vulnerabilidade, como pessoas com doenças crônicas, terminais ou em período de internação, crianças e idosos em processo biológico do envelhecimento ou quadros patológicos, como doenças crônicas, neoplasias, ou doenças neurodegenerativas, sendo o Alzheimer a mais comum.

O cuidado com idosos é complexo, pois o fato dos cuidadores necessitarem responder às exigências de tal função e às solicitações dos outros familiares tem implicações negativas, podendo a própria relação com o idoso que é cuidado ser ambígua e potenciadora de situações desgastantes, que pode ser alavancada se existir relação de parentesco entre os sujeitos, causando sobrecarga pelo acúmulo de papeis (GUERRA HS, et al., 2017; KUCMANSKI LS, et al., 2016; MENDES PN, et al., 2019).

Portanto, cuidar de idoso com Alzheimer é uma tarefa árdua, o cuidador, inúmeras vezes limita a atenção à suas próprias necessidades, em detrimento das exigências cotidianas do cuidar, incluído o conforto, segurança, ajuda em atividades da vida diária (AVD) e as tarefas relacionadas aos afazeres domésticos. Além disso, essa situação é agravada pela falta de informação a respeito da doença, do tratamento utilizado e das estratégias mais adequadas para lidar com os comportamentos problemáticos dos pacientes e o manejo das situações de crise, o que pode provocar consequências negativas (GUERRA HS, et al., 2017; GUTIERREZ LLP, et al., 2017; KUCMANSKI LS, et al., 2016).

No início da doença, esses cuidadores se responsabilizam por atividades mais complexas, como cuidar das finanças desses idosos e fazer compras, e à medida que a doença progride, o cuidado passa a ser mais intenso, tendo que auxiliar em cuidados mais básicos, como por exemplo, higiene pessoal, banho e alimentação, e quando alcança a fase terminal da doença, é exigido ao cuidador cuidados mais específicos, como movimentação total do doente, manuseio de dispositivos (sondas, cateteres e oxigênio), realização de curativos, e até mesmo o conhecimento da situação nutricional e balanço hídrico, uma vez que o idoso se encontra totalmente dependente (MUNIZ EA, et al., 2016). Assim, quanto mais dependente e desfavorável forem as condições dos pacientes, maior será o impacto e as atribuições relativas ao ato de cuidar, desempenhadas pelo cuidador (SEIMA MD, et al., 2014). Poucas pessoas estão preparadas para lidar com a responsabilidade e sobrecarga que é cuidar de um idoso demenciado, pois em geral, existe uma variedade de sentimentos que são despertados nos cuidadores, como o medo, a insegurança, a impotência frente à evolução da doença, a intromissão e a rejeição vinda de outros membros da família, de como agir, 
de como entender a pessoa afetada e seus próprios sentimentos, produzindo desgastes de ordem emocional, física e psicológica para o cuidador e a família (MUNIZ EA, et al., 2016; LEMOS ND, et al., 2006).

As demandas de trabalho dos cuidadores, a aceitação do diagnóstico, o lidar com um estresse cada vez maior, administrar o conflito dentro da família e até mesmo planejar o futuro ocasionam vulnerabilidade a doenças, tensão e sintomas depressivos, que são potencializados quando estes cuidadores são familiares. Fatores como a gravidade da doença, o nível de incapacidade funcional, o comprometimento na habilidade de comunicar-se, o número de medicamentos em uso, a presença de distúrbios de comportamento, visuais, auditivos e/ou cognitivos apresentados pelos pacientes estão associados ao nível de sobrecarga do cuidador. Assim, quanto mais dependente e desfavorável forem as condições dos pacientes, maior será o impacto e as atribuições relativas ao ato de cuidar, desempenhadas pelo cuidador (SEIMA MD, et al., 2014; LEITE BS, et al., 2017b).

Muitas vezes o cuidador vê seu estado de saúde e bem-estar serem afetados e se sente incapaz de enfrentar essa realidade, passando a ser um paciente desconhecido para o serviço de saúde, que requer um diagnóstico precoce e intervenção imediata (GUERRA HS, et al., 2017). Contudo, ser cuidador, também, pode gerar benefícios para quem o executa, tais como a oportunidade de reflexão, autoavaliação, aprendizado, consciência, perdão, criação de vínculo afetivo com o doente e humanização (MARINS AMF e SILVA J, 2017). Seima MD, et al. (2014), Silva MIS, et al. (2018), Kucmanski LS, et al. (2016), Mendes PN, et al. (2019), entre outros, são autores que apontam a necessidade de atenção ao cuidador e a avaliação da sobrecarga relacionada à tarefa dispensada, através do uso de instrumentos específicos e validados, que englobam diversas características que compõem a relação portador-cuidador (GRATÃO ACM, et al., 2013; LINO VTS, et al., 2016; SILVA MIS, et al., 2018).

\section{A Sobrecarga do Cuidador do Portador Alzheimer}

As consequências do Alzheimer, assim como a maioria das enfermidades não se restringem apenas ao portador e abrange diversos atores, dentre eles a família. Essa que, muitas vezes, se encontra nas incertezas do futuro, nas dúvidas não esclarecidas e angústias decorrentes da doença (GARCIA CR, et al., 2017).

Essa situação ocorre, pois na maioria das vezes, o indivíduo dificilmente escolhe ser cuidador, dessa forma essa função é desempenhada por um familiar que não escolheu essa ocupação e que frequentemente é o único que desempenha cuidados domiciliares, ocasionando o autoabandono. Diante disso, cuidadores familiares podem vivenciar durante o desempenho de sua ocupação a sobrecarga, a qual se caracteriza por diversos fatores que levam a deterioração mental, física, emocional e social proporcionando diversas problemáticas no cotidiano desses indivíduos (BAPTISTA BO, et al., 2012; CARDOSO VB, et al., 2017).

Segundo Brigola AG, et al. (2017), diversos fatores associados a função de cuidar e que trazem prejuízos a saúde do cuidador são apontados nas literaturas. Tal processo é analisado por Gratão ACM, et ale (2013), onde é possível notar a relação direta entre aumento da dependência funcional do indivíduo que recebe os cuidados e aumento do nível de sobrecarga do indivíduo que dispensa o cuidado, e também por Costa TG, et al. (2011) que destacam a relação dependência e sobrecarga e aumento do estresse, e frisam que os cuidadores, em sua maioria familiares, auxiliam o portador de Alzheimer em todas as atividades cotidianas. Logo é possível afirmar que a percepção de saúde e a sobrecarga do cuidador são influenciadas pelo tempo de cuidado, o nível de dependência para as atividades cotidianas e o nível de alteração cognitiva do idoso.

Lino VTS, et al. (2016) ao avaliar a prevalência de sobrecarga e fatores associados em cuidadores de idosos em uma região do Rio de Janeiro, constataram o predomínio de sobrecarga alta associada com a falta de apoio e interação social, depressão e aumento de déficit cognitivo em idosos, que aumentam consideravelmente o estresse nos cuidadores. A experiência de assumir a responsabilidade por idosos portadores de Alzheimer tem sido referida por cuidadores como uma tarefa exaustiva e estressante, pelo 
envolvimento afetivo e por ocorrer uma relação de dependência, em que o cuidador, ao desempenhar atividades relacionadas ao bem-estar físico e psicossocial do idoso, passa a ter restrições em relação à sua própria vida (SILVA MIS, et al., 2018).

Esta relação exaustiva também é mencionada por Seima MD, et al. (2014), que constataram em um estudo qualitativo que os cuidadores apresentam-se sobrecarregados e exaustos, em virtude da intensidade de cuidados exigidos pelo idoso com DA, principalmente aqueles em idade avançada, ou seja, dos cuidadores de 60 anos ou mais, um fator decisivo para maiores desgastes destes cuidadores, assim como 0 desenvolvimento de outras atividades associadas ao cuidar. Outras questões como residir com o idoso e o cuidado em tempo prolongado também são determinantes para o aumento da sobrecarga, além da privação da vida social, que também é um grande problema vivenciado pelos cuidadores (NARDI EFR, et al., 2012).

O estudo de Arakaki BK, et al. (2012) demonstrou o perfil socioeconômico dos cuidadores e identificou que a maior parte dos cuidadores são mulheres e possuem idade superior a 60 anos e que a maioria é cônjuge ou filha do portador de Alzheimer. Na mesma pesquisa foram evidenciados sintomas prevalentes nos cuidadores como: ansiedade, agitação, depressão, apatia, desinibição, irritação e delírio. Sintomas que provocam o desgaste e sobrecarga em relação ao cuidado. Em outras pesquisas evidenciou-se que cuidadores com sobrecarga moderada de trabalho decorrente da realização de sua atividade como a falta de descanso ao longo da semana propiciam problemas de coluna e depressão (GUERRA HS, et al., 2017).

Diante desses sintomas é perceptível que ao cuidador familiar são necessários, além das condições de realizar atividades de esforço físico, o equilíbrio emocional e a capacidade de agregar o cuidado com outras atividades, domésticas ou relacionadas às atividades laborais, o que ocasiona uma condição de sobrecarga, a qual compromete o bem-estar físico e mental do cuidador, especialmente se este cuidador for idoso. Dentre as atividades realizadas pelo cuidador que provocam a sobrecargas estão: os diversos tipos de cuidados relacionados ao comprometimento funcional do idoso e às atividades básicas do cotidiano, os quais estão diretamente ligados aos cuidados de manutenção da vida como: "banho, alimentação, vestuário, mobilização e transporte do idoso, e também os relativos à reparação da saúde, como oferecer a medicação" (MARINS AMF e SILVA J, 2017; NARDI EFR, et al., 2012).

O estudo de Araújo CLO, et al. (2012) corrobora com a sobrecarga dos cuidadores. Nessa pesquisa os cuidadores se encontram intensamente vitimados pelo excesso da carga de trabalho pois, após encarregarse desse papel de cuidadores de indivíduos com Alzheimer, não obtém tempo para o meio social ou disposição para se cuidar, de se distrair, com o cansaço corriqueiramente presente. Situação que também é evidenciada por Almeida LPB, et al. (2018), que em sua pesquisa relata o cotidiano das cuidadoras como uma: "rotina de trabalho intensa, ininterrupta e complexa". Além disso destaca falta de opção, ausência de recursos para obter um cuidador profissional e laços estabelecidos entre a pessoa idosa e o cuidador. É importante salientar que a motivação para cuidar pode impactar no cuidado realizado pelos cuidadores e, também, provocar mudanças na qualidade de vida.

É perceptível que a sobrecarga acarreta alterações em vários aspectos da vida do cuidador. Ademais essa sobrecarga indica que os cuidadores necessitam de orientações relacionadas à importância do autocuidado e de suporte dos serviços de saúde para minimizar a sobrecarga de trabalho, doenças provenientes das consequências da sobrecarga. Nessas circunstâncias deve-se promover o desenvolvimento de programas de apoio dos serviços de saúde, os quais devem oportunizar, aos cuidadores instrumentos que facilitam o cotidiano e suporte multiprofissional. (CARDOSO VB, et al., 2017; BAPTISTA BO, et al., 2012).

\section{CONSIDERAÇÕES FINAIS}

Os estudos apontaram que o cuidador se apresenta com uma alta sobrecarga, a qual varia conforme as características do cuidador e do portador da doença, e repercute negativamente na sua saúde mental e física. Evidencia-se, então, a necessidade dos serviços de saúde de se instrumentalizar e disponibilizar recursos para atender esse cuidador por meio de prescrições individualizadas de cuidados, implementação de ações de apoio, capacitação e orientação, as quais considerem o meio biopsicossocial e a valorização 
da relação e do respeito ao ser humano. Este estudo também permitiu verificar a escassez de publicações voltadas para o cuidador do portador de Alzheimer, uma vez que o termo cuidador é amplamente utilizado em literaturas sobre doenças crônicas e condições de vulnerabilidade.

\section{REFERÊNCIAS}

1. ALMEIDA LPB, et al. Características sociais e demográficas de idosos cuidadores e motivos para cuidar da pessoa idosa em domicílio. Revista Mineira de Enfermagem, 2018; 22: 1-7.

2. ARAKAKI BK, et al. Análise do desgaste e da sobrecarga de cuidadores/ familiares de idosos com doença de Alzheimer causado pelos sintomas psicológicos e comportamentais. Revista de Terapia Ocupacional da Universidade de São Paulo, 2012; 23(2): 113-121.

3. ARAÚJO CLO, et al. Perfil de cuidadores de idosos com Doença de Alzheimer. Revista KairósGerontologia, 2012; 15(1): 109-128.

4. BAPTISTA BO, et al. A sobrecarga do familiar cuidador no âmbito domiciliar: uma revisão integrativa da literatura. Revista Gaúcha de Enfermagem, 2012; 33(1): 147-156.

5. BRIGOLA AG, et al. Perfil de saúde de cuidadores familiares de idosos e sua relação com variáveis do cuidado: um estudo no contexto rural. Revista Brasileira de Geriatria e Gerontologia, 2017; 20(3): 410-422.

6. CARDOSO VB, et al. A doença de Alzheimer em idosos e as consequências para cuidadores domiciliares. Memorialidades, 2017; 12(23,24): 113-149.

7. CHAIMOWICZ F. Saúde do Idoso. 2nd ed.Belo Horizonte: NESCON UFMG, 2013; 167p.

8. COSTA TG, et al. Relações familiares no contexto do cuidado à pessoa com Doença de Alzheimer. Revista Enfermagem Integrada, 2011; 4(1): 676-689.

9. DELALIBERA M, et al. Circunstâncias e consequências do cuidar: caracterização do cuidador familiar em cuidados paliativos. Ciência \& Saúde Coletiva, 2018; 23(4): 1105-111.

10. ELIAS CSR, et al. Quando chega o fim? Uma revisão narrativa sobre terminalidade do período escolar para alunos deficientes mentais. SMAD, Revista Eletrônica Saúde Mental Álcool e Drogas (Edição em Português), 2012; 8(1):48-53.

11. GARCIA CR, et al. Cuidadores familiares de idosos com a doença de Alzheimer. Revista Kairós Gerontologia, 2017; 20(1): 409-426.

12. GRATÃO ACM, et al. Dependência funcional de idosos e a sobrecarga do cuidador. Revista da Escola de Enfermagem da USP, 2013; 47(1):137-144.

13. GUERRA HS, et al. A sobrecarga do cuidador domiciliar. Revista Brasileira em Promoção da Saúde, 2017; 30(2): 179-186.

14. GUTIERREZ LLP, et al. Caracterização de cuidadores de idosos da região metropolitana de Porto Alegre (RS): perfil do cuidado. Revista Saúde em Debate, 2017; 41(114): 885-898.

15. IBGE- INSTITUTO BRASILEIRO DE GEOGRAFIA E ESTATíSTICA.2017. In :Número de idosos cresce $18 \%$ em 5 anos e ultrapassa 30 milhões em 2017. Disponível em: https://agenciadenoticias.ibge.gov.br/agencianoticias/2012-agencia-de-noticias/noticias/20980-numero-de-idosos-cresce-18-em-5-anos-e-ultrapassa-30milhoes-em-2017.Acesso em: 15 jan.2020.

16. IBGE: Pesquisa Nacional por Amostra de Domicílios (PNAD) 2017. Disponível em: https://www.ibge.gov.br/estatisticas/multidominio/ciencia-tecnologia-e-inovacao/9127-pesquisa-nacional-poramostra-de-domicilios.html?=\&t=0-que-e. Acesso em: 15 jan. 2020

17. KUCMANSKI LS, et al. Doença de Alzheimer: desafios enfrentados pelo cuidador no cotidiano familiar. Revista Brasileira de Geriatria e Gerontologia, 2016; 19(6): 1022-1029.

18. LEITE BS, et al. Relação do perfil epidemiológico dos cuidadores de idosos com demência e a sobrecarga do cuidado. Cogitare Enfermagem, 2017a; 22(4): 1-11.

19. LEITE BS, et al. A vulnerabilidade dos cuidadores de idosos com demência: estudo descritivo transversal. Revista Brasileira de Enfermagem, 2017b; 70(4):714-720.

20. LEMOS ND, et al. Cuidando do Paciente com Alzheimer: o impacto da doença no cuidador. Saúde e Sociedade, 2006; 15(3): 170-179.

21. LINO VTS, et al. Prevalência de sobrecarga e respectivos fatores associados em cuidadores de idosos dependentes, em uma região pobre do Rio de Janeiro, Brasil. Cadernos de Saúde Pública, 2016; 32(6): 1-14.

22. LUZARDO AR, et al. Características de idosos com Doença de Alzheimer e seus cuidadores: uma série de casos em um serviço de neurogeriatria. Texto\& Contexto Enfermagem, 2006; 15(4): 587-594.

23. MAIA JM, et al. Perfil dos cuidadores de idosos de uma instituição de longa permanência de Imperatriz - MA. Revista Ciência em Extensão, 2018; 14(2): 43-54. 
24. MARINS AMF, SILVA J. O impacto do comportamento do idoso com doença de Alzheimer na vida do cuidador. Revista de Enfermagem do Centro-Oeste Mineiro, 2017; 7: 1-7.

25. MENDES PN, et al. Sobrecargas física, emocional e social dos cuidadores informais de idosos. Acta Paulista de Enfermagem, 2019; 32(1): 87-94.

26. MUNIZ EA, et al. Grau de sobrecarga dos cuidadores de idosos atendidos em domicílio pela Estratégia Saúde da Família.Revista Saúde em Debate, 2016; 40(110): 172-182.

27. NARDI EFR, et al. Dificuldades dos cuidadores familiares no cuidar de um idoso dependente no domicílio. Ciência, cuidado e saúde, 2012; 11(1): 98-105.

28. ORGANIZAÇÃO DAS NAÇÕES UNIDAS (THE UNITED NATIONS). 2019. In: World Population Prospects 2019 : Highlights. Disponível em: https://www.un.org/development/desa/publications/world-population-prospects-2019highlights.html. Acesso em: 15 jan. 2020.

29. SEIMA MD, et al. Relação no cuidado entre o cuidador familiar e o idoso com Alzheimer. Revista Brasileira de Enfermagem, 2014; 67(2): 233-240.

30. SILVA MIS, et al. Doença de Alzheimer: repercussões biopsicossociais na vida do cuidador familiar. Revista de Enfermagem UFPE On Line, 2018; 12(7): 1931-1939.

31. XIMENES MA, et al. Doença de Alzheimer: a dependência e o cuidado. Revista Kairós Gerontologia, 2014; 17(2): 121-140. 